\title{
ABSTRACT WAVE EQUATIONS WITH FINITE VELOCITY OF PROPAGATION ${ }^{1}$
}

\author{
BY STEPHEN BERMAN
}

Communicated by Ray A. Kunze, June 22, 1971

Let $G$ be a locally compact abelian group. Let $L^{2}(G)$ be the Hilbert space of all complex-valued functions on $G$ that are measurable and square-integrable with respect to Haar measure on $G$. Let $B$ be a selfadjoint translation-invariant operator in $L^{2}(G)$, not necessarily bounded, and consider the abstract wave equation

$$
d^{2} u / d t^{2}+B^{2} u=0
$$

where $u$ is a function from the nonnegative real axis to $L^{2}(G)$. For any $\phi$ and $\psi$ in $L^{2}(G)$, a solution is given by

$$
u(t)=(\cos t B) \phi+\left(\frac{\sin t B}{B}\right) \psi
$$

in the sense that

$$
\frac{d^{2}}{d t^{2}}\langle u(t), w\rangle+\left\langle u, B^{2} w\right\rangle=0
$$

for all $w$ in the domain of $B^{2}$, where $\langle\cdot, \cdot\rangle$ denotes the usual inner product in $L^{2}(G)$. In the classical case in which $G$ is an $n$-dimensional Euclidean space $R^{n}$ and $-B^{2}$ is the Laplacian, the solution $\left(^{*}\right)$ has the property that if $\phi$ and $\psi$ have compact support then so does $u(t)$ for all $t>0$. In fact, there exists a compact subset $K_{t}$ of $R^{n}$, independent of $\phi$ and $\psi$, such that supp $u(t) \subset(\operatorname{supp} \phi \cup \operatorname{supp} \psi)+K_{t}$, where $\operatorname{supp} f$ denotes the support of $f$. Our first theorem says that on $\boldsymbol{R}^{n}$ this is essentially the only operator $B$ for which the abstract wave equation has this property, which we call finite velocity of propagation. Recall now that if $B$ is a selfadjoint translation-invariant operator in $L^{2}(G)$, then there must exist a real measurable function $\beta$ on the dual group $\Gamma$ of $G$ such that $(B f)^{\wedge}=\beta \hat{f}$, where $\hat{f}$ denotes the Fourier transform of $f$.

A MS 1970 subject classifications. Primary 22B99, 34G05, 35B30, 35L05, 47B25.

Key words and phrases. Wave equation, locally compact abelian group, translationinvariant operators, Laplacian, velocity of propagation, distributions on an LCA group.

1 Research was done while the author held an NSF Graduate Fellowship. 
THEOREM 1. Let $B$ be a selfadjoint translation-invariant operator in $L^{2}(G)$, with $\beta$ as above.

(a) Suppose $G=R^{n}$. Then the following are equivalent:

(i) $\beta^{2}$ is a second degree polynomial (so that $B^{2}$ is a second order differential operator);

(ii) there exist functions $s_{1}(t), s_{2}(t), \cdots, s_{n}(t)$ on $0 \leqq t<\infty$ such that each $s_{j}(j=1, \cdots, n)$ is bounded on some interval containing 0 and such that if $\phi \in L^{2}\left(R^{n}\right)$ and

$$
\operatorname{supp} \phi \subset\left\{x=\left(x_{1}, \cdots, x_{n}\right) \in R^{n}:\left|x_{j}\right| \leqq a_{j}(j=1, \cdots, n)\right\}
$$

then

$$
\operatorname{supp}((\cos t B) \phi) \subset\left\{x \in R^{n}:\left|x_{j}\right| \leqq a_{j}+s_{j}(t)(j=1, \cdots, n)\right\} .
$$

If (i) is satisfied and $\beta(x)^{2}=\sum_{i, j=1}^{n} a_{i j} x_{i} x_{j}+$ lower order terms, then in (ii) we may take $s_{j}(t)=t \sqrt{ } a_{j j}(j=1, \cdots, n)$, and the operator ( $\sin t B) / B$ has the same property as $\cos t B$ in (ii).

(b) Suppose $\Gamma$ does not contain $R$ as a subgroup. Then the following are equivalent:

(i) there is a compact open subgroup $\Lambda$ of $\Gamma$ such that $\beta$ is constant on cosets of $\Lambda$;

(ii) there exists, for each $t \geqq 0$, a compact subset $K_{t}$ of $G$ such that supp $((\cos t B) \phi) \subset \operatorname{supp} \phi+K_{t}$ for any $\phi \in L^{2}(G)$ with compact support and such that the closure of $\cup\left\{K_{t}: 0 \leqq t \leqq t_{0}\right\}$ is compact for some $t_{0}>0$.

If (ii) is satisfied, then there is a compact open subgroup $H$ of $G$ containing all the $K_{t}$ and we may take $\Lambda$ to be the annihilator of $H$. The operator $\cos t B$ is the convolution operator $\phi \rightarrow \phi * \mu_{t}$ where $\mu_{t}$ is a distribution on $G$ in the sense of Bruhat [1].

By combining (a) and (b) with the structure theorem for abelian groups, one can obtain a description of all $B$ such that $\left(^{*}\right)$ has finite velocity of propagation.

So far we have only demanded the existence of compact $K_{t}$. From Theorem 1a, we see that in $R^{n}, K_{t}$ is small for small $t$. This is not true in general. For compact $G$, any $\beta$ will satisfy Theorem $1 \mathrm{~b}$, but the assumption that $K_{t}$ be small for small $t$ restricts the possibilities for $\beta$. For example, on the torus we have the following theorem:

Theorem 2. Let $G$ be the one-dimensional torus, and let $\beta$ and $\mu_{t}$ be as in Theorem 1. Then the following are equivalent:

(i) for any neighborhood $U$ of 0 in $G$, supp $\mu_{t} \subset U$ for $t$ sufficiently small;

(ii) $\beta^{2}$ is the restriction to the integers of a second degree polynomial that is positive on all of $\boldsymbol{R}$. 
These results are contained in a thesis to be submitted in partial fulfillment of the requirements for the Ph.D. at the Massachusetts Institute of Technology. Complete proofs will appear elsewhere. The author would like to thank Professor I. E. Segal for suggesting this line of investigation and for many helpful conversations.

\section{REFERENCES}

1. F. Bruhat, Distributions sur une groupe localement compact et applications à l'étude des représentations des groupes p-adiques, Bull. Math. Soc. France 89 (1961), 43-75. MR 25 \#4354.

Massachusetts Institute of Technology, Cambridge, Massachusetts 02139 